\title{
Screening For Unknown Compounds in Forensic Toxicology
}

\author{
Muhammad Imran \\ Forensic Toxicology Department \\ Punjab Forensic Science Agency \\ Lahore 53700, Pakistan \\ imranfstox@gmail.com
}

\begin{abstract}
The purpose of postmortem forensic toxicological analysis is to determine the role of any unknown drug/poison in causing death. The first and the most important step in the analysis scheme is the screening of biological specimens for the presence of drugs/poison. Currently, general unknown screening (GUS) or systemic toxicological analysis (STA) procedure involves gas chromatography-mass spectrometry $(G C-M S)$. However, non volatile or thermolabile compounds cannot be accurately analyzed on GC-MS. Therefore liquid chromatography with mass detector (LC-MS) could complement GC-MS for unknown drug screen. Both GC and LC techniques have inherent limitations that prevent the detection of certain types of compounds. Recent developments in analytical techniques especially in triple quad, ion trap and time of flight mass spectrometers have improved the sensitivity and specificity for targeted and untargeted screening of drugs, pesticides and other relevant compounds.
\end{abstract}

Keywords: Toxicological analysis, unknown screen, drugs, poisons, analytical techniques.

Identification and quantitaion of unknown toxic chemicals including drugs, drugs of abuse, poisons and/or their metabolites in biological matrices is a major part of analysis in forensic toxicology. This type of analysis is known as systematic toxicological analysis (STA) or general unknown screenings (GUS). Analytical methods for STA should ideally cover all relevant drugs, poisons and their metabolites and allow unambiguous identification of poisonous compounds [1-3]. Generally gas chromatography-mass spectrometry (GC-MS) is applied for screening and confirmation analysis in STA cases [4]. It is considered as gold standard because the availability of uniform mass spectra libraries which are encompassing thousands of forensically important compounds. However, non volatile or thermolabile compounds cannot be accurately analyzed on GC-MS. Therefore a trend has shifted to fill the gap with respect to thermolabile and non-volatile analytes towards liquid chromatography-mass spectrometry (LC-MS) to assist existing GC-MS procedures. One such approach for untargeted screening analysis of poisons makes use of LC-MS/MS under data-dependent acquisition control (DDA) to identify compounds by subsequent library search [5,6]. Now many classes of drugs, poisons including pesticides and anticoagulant rodenticides are being analyzed on LC-MSMS [7-11].

Recently published LC-MS-based screening procedures for STA analysis can be divided into two general approaches: In the first approach, triple quadrupole and ion trap mass spectrometers are used to produce product ion spectra which can be searched against reference mass spectra libraries which were build from previously recorded mass spectra on the same or similar type of apparatus. More than 700 toxicologically important compounds have been detected using triple quadrupole or ion trap mass spectrometers $[8,9]$. This approach is relatively more specific for targeted screen of drugs/poison. The second approach uses high-resolution mass spectrometry with benchtop time-of-flight (TOF) mass spectrometers, where compounds are identified by searching the accurate masses in the sample with accurate mass databases of toxicologically relevant compounds. Recently time-of-flight (TOF) mass spectrometer based methods have detected approximately 50500 compounds in blood, urine and hair specimens $[10,11]$. This approach has additional advantages for untargeted screen of compounds.

LC-MS/MS has become increasingly important in forensic toxicology especially for multi-target screening and/or quantification of drugs, poisons and their metabolites in conventional and alternative matrices. However, LC-MS reference libraries cannot be transferred between different instrument types. The benchtop TOF-MS instruments have provided an alternative screening approach based on high-resolution MS which at least theoretically could cover compounds for which no reference standards are available. Currently the adaptability of this approach in routine analysis is hindered by 
the problem of discriminating between isomeric compounds. The development of benchtop orbitrap mass spectrometers has further unlocked the option to combine information-rich mass spectra and high-resolution MS in a single approach [12].

The advancements in analytical techniques cannot overcome the problem of complex biological matrices; therefore good sample preparations methods can complement these techniques in unraveling unknown drugs and poisons. Traditionally liquid-liquid extraction and solid phase extraction have been used for extracting drugs from various biological specimens including blood, urine, liver and hair $[13,14]$. Recently many modifications have been made in the extraction techniques to further improve the sensitivity and recovery of analytes from biological matrices. For instance solid phase microextraction, matrix solid phase dispersion, accelerated solvent extraction, supercritical fluid extraction, hollow fiber liquid phase microextraction and dispersive liquid-liquid microextraction have been introduced for this purpose.

\section{CONCLUSION}

GC-MS has found extensive use in the screening of unknown compounds in forensic toxicology because of availability universal spectra libraries for thousands of toxicologically relevant compounds however for thermolabile drugs/poisons; LC-MS or LC with triple quad/ion trap/QTOF is considered a better technique. For solving STA cases with minimum uncertainty, it is likely that some combination of improved analytical techniques and extraction procedures along with software enhancements to translate LC-MS mass spectra into uniform database will be possible in future. For the complete screen of unknown compounds, both GC-MS and LC-MS/MS would give more wide range of analytes which could be identified and quantitated.

\section{REFERENCES}

[1] Maurer H.H., Position of chromatographic techniques in screening for detection of drugs or poisons in clinical and forensic toxicology and/or doping control, Clin. Chem. Lab. Med. 42, 1310-24 (2004).

[2] Skelton H., Dann L.M., Ong R.T., Hamilton T. and Ilett K.F., Drug screening of patients who deliberately harm themselves admitted to the emergency department, Ther. Drug Monit. 20, 98103 (1998).

[3] Wu A.H., McKay C., Broussard L.A., Hoffman R.S., Kwong T.C., Moyer TP, Otten E.M., Welch S.L. and Wax P., National academy of clinical biochemistry laboratory medicine practice guidelines: recommendations for the use of laboratory tests to support poisoned patients who present to the emergency department. Clin. Chem. 49, 357-79 (2003).

[4] Maurer H.H., Hyphenated mass spectrometric techniques-indispensable tools in clinical and forensic toxicology and in doping control, J. Mass Spectrom. 41, 1399-1413 (2006).

[5] Peters F.T., Recent advances of liquid chromatography-(tandem) mass spectrometry in clinical and forensic toxicology, Clin. Biochem. 44, 54-65 (2011).

[6] Oberacher H., Schubert B., Libiseller K. and Schweissgut A., Detection and identification of drugs and toxicants in human body fluids by liquid chromatography-tandem mass spectrometry under data-dependent acquisition control and automated database search, Anal. Chim. Acta. 770, 121-131(2013).

[7] Imran, M., Shafi, H., Wattoo, S.A., Chaudhary, M.T. and Usman, H.F., Analytical methods for determination of anticoagulant rodenticides in biological samples. Forensic science international, 253, 94-102 (2015).

[8] Dresen S., Ferreiros N., Gnann H., Zimmermann R. and Weinmann W., Detection and identification of 700 drugs by multi-target screening with a 3200 Q Trap (R) LC-MS/MS system and library searching, Anal. Bioanal. Chem. 396, 2425-34 (2010).

[9] Liu H.C., Liu R.H., Ho H.O. and Lin D.L., Development of an information-rich LC-MS/MS database for the analysis of drugs in postmortem specimens, Anal. Chem. 81, 9002-11(2009).

[10] Polettini A., Gottardo R., Pascali J.P. and Tagliaro F., Implementation and performance evaluation of a database of chemical formulas for the screening of pharmaco/toxicologically relevant compounds in biological samples using electrospray ionization-time-of-flight mass spectrometry, Anal. Chem. 80, 3050-7 (2008). 
[11] Liotta E., Gottardo R., Bertaso A. and Polettini A., Screening for pharmaco-toxicologically relevant compounds in biosamples using high-resolution mass spectrometry: a 'metabolomic' approach to the discrimination between isomers, J. Mass Spectrom. 45, 261-71(2010).

[12] Bateman K.P., Kellmann M., Muenster H., Papp R. and Taylor L., Quantitative-qualitative data acquisition using a benchtop orbitrap mass spectrometer, J. Am. Soc. Mass Spectrom. 20, 144150 (2009).

[13] Imran, M., Ashiq, M.Z., Shafi, H., Usman, H.F., Wattoo, S.A., Sarwar, M. and Tahir, M.A., Hair analysis of an unusual case of Chloroquine intoxication. Legal Medicine. 19, 5-10 (2016).

[14] Johnson, R.D. and Botch, S.R., The screening of forensic blood, urine, and tissue specimens for xenobiotics using ion-trap liquid chromatography-tandem mass spectrometry. Journal of analytical toxicology. 35, 65-74 (2011). 